\title{
Hermitian symplectic geometry and extension theory*
}

\author{
M. Harmer
}

\begin{abstract}
Here we give brief account of hermitian symplectic spaces, showing that they are intimately connected to symmetric as well as self-adjoint extensions of a symmetric operator. Furthermore we find an explicit parameterisation of the Lagrange Grassmannian in terms of the unitary matrices $\mathrm{U}(n)$. This allows us to explicitly describe all self-adjoint boundary conditions for the Schrödinger operator on the graph in terms of a unitary matrix. We show that the asymptotics of the scattering matrix can be simply expressed in terms of this unitary matrix.
\end{abstract}

\section{Introduction}

The main motivation to study hermitian symplectic spaces - this terminology follows [10]-is the well know connection between the self-adjoint extensions of a symmetric operator and the Lagrange planes of a hermitian symplectic space [13, 10, 12. This is based on the fact that the boundary form of a symmetric operator is a hermitian symplectic form and the extensions of the operator may be identified with isotropic subspaces in the associated hermitian symplectic space.

In the first section of this paper we define and describe some of the properties of hermitian symplectic spaces. By our definition hermitian symplectic spaces (unlike symplectic spaces) need not be even dimensional or admit a canonical basis. We show that when a hermitian symplectic space admits a canonical basis, it has Lagrange planes and derive an explicit parameterisation of the set of Lagrange planes in terms of the set of unitary matrices $\mathrm{U}(n)$ where $n$ is half the dimension of the space.

In the following section we consider connections to extension theory of symmetric operators. It is observed that hermitian symplectic spaces that do not admit a canonical basis, or Lagrange planes, correspond to symmetric operators with unequal deficiency indices (in this case the extensions are described by isotropic subspaces). On the other hand, symmetric operators with equal deficiency indices correspond to hermitian symplectic spaces with Lagrange planes and as is well known these Lagrange planes may be used to describe the self-adjoint extensions. The fact that the set of Lagrange planes, or self-adjoint extensions, is isomorphic to $\mathrm{U}(n)$ is in accordance with

*AMS: Primary 47A20; Secondary 34B45, 34L40, 47A40, 81U20 
the parameterisation of self-adjoint extensions by a unitary map between the deficiency subspaces as described by Neumann extension theory [2].

We then consider the specific example of the Schrödinger operator on the graph. Our explicit parameterisation of the Lagrange planes in terms of the unitary matrices allows us to describe all self-adjoint boundary conditions at the origin for the Schrödinger operator on the graph with trivial compact part in terms of a unitary matrix. Furthermore, we show that the asymptotics of the scattering matrix may be written in terms of this unitary matrix and that the boundary conditions do not contribute to the discrete spectrum iff this unitary matrix is also hermitian. We also use a property of this parameterisation, as well as the Wronskian, to show the unitarity of the scattering matrix.

\section{Hermitian symplectic geometry}

Many of the basic ideas in this section can be found in any standard text on symplectic geometry [4, 5, 7, 11. However, the concept of a canonical hermitian symplectic space and the details of the parameterisation of Lagrange planes in a hermitian symplectic space distinguish this construction from the standard symplectic case. In particular the Lagrange planes in hermitian symplectic geometry are parameterised by unitary matrices whereas they have different parameterisations in the standard symplectic geometry. Also, by our definition, a hermitian symplectic space need not be even dimensional or admit a canonical basis - unlike the symplectic case. This is seen to correspond to a symmetric operator with unequal deficiency indices.

Definition 1 The two-form $\langle\cdot, \cdot\rangle$, linear in the second argument and conjugate linear in the first argument, is a hermitian symplectic form if

$$
\langle\phi, \psi\rangle=-\overline{\langle\psi, \phi\rangle} .
$$

We recall that the standard symplectic form obeys $\langle\phi, \psi\rangle=-\langle\psi, \phi\rangle$. We will use the prefix 'hermitian' to emphasise this distinction.

Definition 2 We say that an $m$-dimensional $(m<\infty)$ vector space $H_{m}$ over $\mathbb{C}$ is a hermitian symplectic space if it has defined on it a nondegenerate hermitian symplectic form. By nondegenerate we mean that if $\phi$ obeys

$$
\langle\phi, \psi\rangle=0 \quad \forall \psi \in H_{m}
$$

then $\phi=0$.

Since $H_{m}$ is a vector space we can find a basis $\left\{e_{i}\right\}_{i=1}^{m}$ for it and use this basis to express the hermitian symplectic form as a matrix with entries

$$
\omega_{i j}=\left\langle e_{i}, e_{j}\right\rangle .
$$

By the definition of the form, the matrix $\omega$ is a skew-hermitian, $\omega=-\omega^{\star}$, nondegenerate matrix. Clearly the hermitian symplectic form can be written

$$
\langle\phi, \psi\rangle=(\phi, \omega \psi)
$$


where, on the right hand side, $\phi$ and $\psi$ are written as vectors in $\mathbb{C}^{m}$ using the basis $\left\{e_{i}\right\}_{i=1}^{m}$ and $(\cdot, \cdot)$ is the standard hermitian scalar product on $\mathbb{C}^{m}$, making it an $m$-dimensional Hilbert space.

In the usual symplectic case $\omega$ is skew-symmetric and hence, due to nondegeneracy, of even order. This restriction does not apply to skew-hermitian matrices and hence there is no obstruction to having hermitian symplectic spaces of odd dimension.

Hermitian symplectic spaces differ from symplectic spaces in another important respect; given any symplectic space it is always possible to find a canonical basis:

Definition 3 A basis $\left\{p_{i}, q_{i}\right\}_{i=1}^{n}$ which has the following property

$$
\begin{array}{r}
\left\langle p_{i}, q_{j}\right\rangle=\delta_{i j}=-\left\langle q_{j}, p_{i}\right\rangle \\
\left\langle p_{i}, p_{j}\right\rangle=0=\left\langle q_{i}, q_{j}\right\rangle
\end{array}
$$

where $\delta_{i j}$ is the Kronecker delta is known as a canonical basis.

Even an even-dimensional hermitian symplectic space, $H_{2 n}$, need not admit a canonical basis. Let us suppose that $H_{2 n}$ has a basis $\left\{e_{i}\right\}_{i=1}^{2 n}$ so that the skew-hermitian matrix $\omega$ is

$$
\omega=\left(\left\langle e_{i}, e_{j}\right\rangle\right)=i \mathbb{I}_{(2 n)} .
$$

We denote by $\mathbb{I}$ or $\mathbb{I}_{(n)}$ the $n \times n$ unit matrix. This case is obviously prohibited in the symplectic case but acceptable in the hermitian symplectic case. Now if it were possible to find a canonical basis in this space then there would be a non-singular transformation of the basis, $P$, such that $\omega$ would be transformed to

$$
P^{\star} \omega P=J
$$

where $J$, known as the canonical symplectic structure, is

$$
J=\left(\begin{array}{cc}
0 & \mathbb{I} \\
-\mathbb{I} & 0
\end{array}\right) .
$$

This is clearly not possible. We use the fact that any non-singular transformation can be written as the product of a unitary and a hermitian matrix, $P=U H$. Consequently

$$
i H^{2}=P^{\star} \omega P=J
$$

and the left hand side is a matrix with eigenvalues only on the imaginary axis in the upper half plane. The right hand side, $J$, however, has eigenvalues $\pm i$ equally distributed between the upper and lower half planes.

Definition 4 We say that a hermitian symplectic space is canonical if it admits a canonical basis.

In the following we denote

$$
\mathbb{I}_{\left(n_{+}, n_{-}\right)} \equiv\left(\begin{array}{cc}
\mathbb{I}_{\left(n_{+}\right)} & 0 \\
0 & -\mathbb{I}_{\left(n_{-}\right)}
\end{array}\right) .
$$


Lemma 1 A hermitian symplectic space $H_{m}$ is, up to a non-singular transformation of the basis, completely characterised by two integers, $n_{+}, n_{-}$, $n_{+}+n_{-}=m$. Specifically the matrix $\omega$ associated with the hermitian symplectic form can be diagonalised to

$$
i \mathbb{I}_{\left(n_{+}, n_{-}\right)} .
$$

Furthermore $H_{m}$ is canonical iff $n_{+}=n_{-}$.

Proof: A hermitian symplectic space is specified by the matrix $\omega$ up to a non-singular transformation of the basis, $P$. The matrix $-i \omega$ is hermitian and hence it can be diagonalised

$$
-i \omega=U D U^{\star}
$$

where $D$ is a real diagonal matrix without zeroes on the diagonal. Let us choose the matrix $H$ as the positive diagonal matrix so that $D^{2}=H^{4}$. Then choosing the non-singular transformation of the basis, $P=U H^{-1}$ we get

$$
P^{\star} \omega P=i H^{-1} U^{\star} U D U^{\star} U H^{-1}=i \mathbb{I}_{\left(n_{+}, n_{-}\right)}
$$

where $n_{ \pm}$are the number of positive and negative eigenvalues of $-i \omega$ respectively. Clearly, when $n_{+}=n_{-}=n$ we can find a canonical basis since we can transform $i \mathbb{I}_{(n, n)}$ to $J$.

Definition 5 We say that $\phi, \psi \in H_{m}$ are skew-orthogonal, denoted $\phi \perp \psi$, if

$$
\langle\phi, \psi\rangle=0 .
$$

Definition 6 Given a subspace $N \subset H_{m}$, we define the skew-orthogonal complement, $N^{\perp}$, as the subspace

$$
N^{\perp} \equiv\left\{\phi ; \phi \in H_{m},\langle\phi, \psi\rangle=0 \forall \psi \in N\right\} .
$$

Definition 7 The subspace $N \subset H_{m}$ is isotropic if

$$
N \subset N^{\perp} .
$$

Let us assume that we have fixed some basis and found the corresponding skew-hermitian matrix $\omega$ from equation (11) so that $H_{m}$ can be identified with the Hilbert space $\mathbb{C}^{m}$ equipped with a hermitian symplectic form. The remaining lemmata in this section all have analagous statements in symplectic geometry [7, 11].

Lemma 2 The subspace $N \subset H_{m}$ is isotropic iff the subspaces $N$ and $\omega N$ are orthogonal in $\mathbb{C}^{m}$.

Proof: Follows directly from equation (2).

Lemma 3 The dimension, $k$, of an isotropic subspace $N \subset H_{m}$ never excedes $m / 2$. 
Proof: Since the operator $\omega$ on $\mathbb{C}^{m}$ is nondegenerate, the dimensions of $N$ and $\omega N$ are the same. Consequently $k+k \leq m$.

Definition 8 An isotropic subspace $\Pi_{n} \subset H_{2 n}$ of maximal dimension, that is dimension $n$, is called a Lagrange plane.

Corollary 1 If $\Pi_{n} \subset H_{2 n}$ is a Lagrange plane then $\Pi_{n}^{\perp}=\Pi_{n}$.

Proof: $\Pi_{n}$ and $\Pi_{n}^{\perp}$ both have dimension $n$ and $\Pi_{n} \subset \Pi_{n}^{\perp}$.

From the definition it is clear that Lagrange planes only exist in evendimensional hermitian symplectic spaces, in fact it is not difficult to show that a hermitian symplectic space contains a Lagrange plane iff it is canonical. First we need the basic lemma,

Lemma 4 Given a hermitian symplectic subspace $V \subset H_{m}, V^{\perp}$ is also hermitian symplectic,

$$
V+V^{\perp}=H_{m}
$$

and these subspaces have trivial intersection.

Proof: It is clear that the intersection $V \cap V^{\perp}$ is empty. Supposing instead that there is a $v \in V \cap V^{\perp}$ then $v$ is skew-orthogonal to all the elements of $V$ and hence the form is degenerate on $V$ which is a contradiction.

Since the matrix $\omega_{i j}$ is nondegenerate the dimension of $V^{\perp}$ is the codimension of $V$. But since these two spaces do not intersect, by a simple argument of linear independence

$$
V+V^{\perp}=H_{m}
$$

Now we suppose that the form is degenerate on $V^{\perp}$, so there is some element $z \in V^{\perp}$ so that

$$
\langle z, u\rangle=0, \quad \forall u \in V^{\perp}
$$

and

$$
\langle z, v\rangle=0, \quad \forall v \in V .
$$

But this would imply that the form is degenerate on $H_{m}$ which is a contradiction.

Lemma 5 An even-dimensional hermitian symplectic space $H_{2 n}$ is canonical iff it contains a Lagrange plane.

Proof: It is clear that a canonical hermitian symplectic space contains a Lagrange plane, viz. the span of the first $n$ elements of the canonical basis. We suppose that we have an even-dimensional hermitian symplectic space $H_{2 n}$ which contains a Lagrange plane $\Pi_{n}$. Then we can find some basis $\left\{e_{i}\right\}_{i=1}^{2 n}$ so that the first $n$ elements $\operatorname{span} \Pi_{n}$. Let us pick $p_{1}=e_{1}$. Since the form is nondegenerate, there is an element $\hat{q}_{1} \notin \Pi_{n}$ such that $\left\langle p_{1}, \hat{q}_{1}\right\rangle \neq 0$ and hence we can normalise so that

$$
\left\langle p_{1}, q_{1}\right\rangle=1 .
$$


We denote by $V_{1}$ the linear span of $\left\{p_{1}, q_{1}\right\}$. Using the fact that $\left\langle p_{1}, p_{1}\right\rangle=0$ it is not difficult to see that $V_{1}$ is a canonical hermitian symplectic space. Applying lemma 4 to $V_{1}$ we see that $V_{1}^{\perp}$ is a hermitian symplectic space. Furthermore it has a Lagrange plane given by the span of $\left\{e_{i}\right\}_{i=2}^{n}$. Repeating this process for $V_{1}^{\perp}$ allows us to construct a canonical basis for $H_{2 n}$.

Definition 9 A linear transformation is called $J$-unitary or hermitian symplectic if it satisfies

$$
g^{\star} J g=J .
$$

Clearly such a transformation takes Lagrange planes to Lagrange planes. Consider the set of all Lagrange planes of a canonical hermitian symplectic space $H_{2 n}$, the Lagrange Grassmannian denoted $\Lambda_{n}$. We show that the Lagrange Grassmannian is isomorphic to the set of unitary matrices.

Lemma 6 A given Lagrange plane $\Pi_{0, n}$ can be made to coincide with any other Lagrange plane $\Pi_{n}$ by means of a hermitian symplectic transformation of the form

$$
g=\left(\begin{array}{cc}
A & B \\
-B & A
\end{array}\right) \quad A, B \in \mathbb{C}^{n \times n}
$$

where $A$ and $B$ satisfy

$$
\begin{gathered}
A^{\star} A+B^{\star} B=\mathbb{I} \\
A^{\star} B=B^{\star} A .
\end{gathered}
$$

Specifically, if we are given a canonical basis $\left\{\xi_{0, i}\right\}_{i=1}^{2 n}$, the first $n$ elements of which span the Lagrange plane $\Pi_{0, n}$, then there is a hermitian symplectic transformation $g$ such that the first $n$ elements of the canonical basis $\left\{\xi_{i}\right\}_{i=1}^{2 n}$ given by

$$
\xi_{i}=\sum_{j=1}^{2 n} g_{i j} \xi_{0, j}
$$

$\operatorname{span} \Pi_{n}$.

Proof: As we are dealing with canonical spaces there always exists a canonical basis $\left\{\xi_{0, i}\right\}_{i=1}^{2 n}$ and we choose $\Pi_{0, n}$ to be the span of the first $n$ elements of this basis. In terms of this canonical basis we can identify $H_{2 n}$ with $\mathbb{C}^{2 n}$ where the two-form is given by $\omega=J$.

Consider another arbitrary Lagrange plane $\Pi_{n}$. Using the above identification, $\Pi_{n}$ may be considered to be an $n$-dimensional subspace of $\mathbb{C}^{2 n}$. Consequently, we can find a set of $n$ orthonormal vectors in $\mathbb{C}^{2 n}$ which form a basis for $\Pi_{n}$-we denote this basis by $\left\{\xi_{i}\right\}_{i=1}^{n}$. Since the $\left\{\xi_{0, i}\right\}$ form a basis for $H_{2 n}$ there are matrices $A$ and $B$ such that

$$
\xi_{i}=\sum_{j}^{n} A_{i j} \xi_{0, j}+\sum_{j}^{n} B_{i j} \xi_{0, j+n} \quad \text { for } i=1, \ldots, n .
$$

That is $A_{i j}=\left(\xi_{i}, \xi_{0, j}\right), B_{i j}=\left(\xi_{i}, \xi_{0, n+j}\right)$ for $j=1, \ldots, n$. Furthermore, since we have assumed that the $\left\{\xi_{i}\right\}$ are orthonormal in $\mathbb{C}^{2 n}$ we immediately have equation (4). Using the fact that the $\left\{\xi_{i}\right\}$ form a Lagrange plane 
in equation (2) gives us equation (5). Together these two equations imply that $g$ is a hermitian symplectic transformation.

In fact, it is easy to see that equations (415) imply that $g$ is a hermitian symplectic matrix as well as a unitary matrix, ie. it preserves the hermitian symplectic form as well as the scalar product in $\mathbb{C}^{2 n}$.

Let us denote by $\mathcal{G}$ the set of matrices of the form

$$
\mathcal{G}=\left\{g=\left(\begin{array}{cc}
A & B \\
-B & A
\end{array}\right) ; A, B \in \mathbb{C}^{n \times n}, g \in \mathrm{U}(2 n)\right\}
$$

which occur in the above lemma, this set is clearly a group under matrix multiplication. In order to classify $\Lambda_{n}$ we need to find the stationary subgroup of $\mathcal{G}$, ie. $\mathcal{H} \subset \mathcal{G}$ the elements of which take the Lagrange plane $\Pi_{0, n}$ into itself. But it is easy to see that in the notation of the above lemma these are just those matrices with $B=0$ : the stationary subgroup $\mathcal{H}$ is therefore the set of matrices

$$
\mathcal{H}=\left\{h=\left(\begin{array}{cc}
C & 0 \\
0 & C
\end{array}\right) ; C \in \mathbb{C}^{n \times n}, h \in \mathrm{U}(2 n)\right\} .
$$

Lemma 7 The Lagrange Grassmannian $\Lambda_{n}$ is in one-to-one correspondence with the unitary group.

$$
\Lambda_{n} \simeq \mathcal{G} / \mathcal{H} \simeq \mathrm{U}(n)
$$

Proof: The first isomorphism follows from lemma 6. To see the second isomorphism we use the unitary matrix

$$
W=\frac{1}{\sqrt{2}}\left(\begin{array}{cc}
\mathbb{I} & i \mathbb{I} \\
i \mathbb{I} & \mathbb{I}
\end{array}\right)
$$

Our choice of $W$ is motivated by the fact that it diagonalises in the 'blockwise' sense matrices of the form given by equation (31). Precisely

$$
W g W^{\star}=W\left(\begin{array}{cc}
A & B \\
-B & A
\end{array}\right) W^{\star}=\left(\begin{array}{cc}
A-i B & 0 \\
0 & A+i B
\end{array}\right) .
$$

Since $g$ is unitary so is $W g W^{\star}$ and hence, $A-i B$ and $A+i B$ must also be unitary.

Now instead of considering the groups $\mathcal{G}$ and $\mathcal{H}$, we consider the unitarily equivalent groups

$$
\hat{\mathcal{G}}=W \mathcal{G} W^{\star}=\left\{\hat{g}=\left(\begin{array}{cc}
S & 0 \\
0 & T
\end{array}\right) ; S, T \in \mathrm{U}(n)\right\}
$$

and, since the elements of $\mathcal{H}$ are already in block diagonal form,

$$
\hat{\mathcal{H}}=W \mathcal{H} W^{\star}=\left\{\hat{h}=\left(\begin{array}{cc}
C & 0 \\
0 & C
\end{array}\right) ; C \in \mathrm{U}(n)\right\} .
$$


It is easy to see that we can represent the set of cosets $\hat{\mathcal{G}} / \hat{\mathcal{H}}$ by the subgroup of $\hat{\mathcal{G}}$ consisting of matrices where the bottom right block is of the form $T=\mathbb{I}$, that is

$$
\Lambda_{n} \simeq \hat{\mathcal{G}} / \hat{\mathcal{H}} \simeq\left\{\hat{g}=\left(\begin{array}{cc}
U & 0 \\
0 & \mathbb{I}
\end{array}\right) ; U \in \mathrm{U}(n)\right\}
$$

This gives the result.

Corollary 2 A given Lagrange plane can be made to coincide with any other Lagrange plane by means of a hermitian symplectic transformation of the form

$$
g=W^{\star} \hat{g} W=W^{\star}\left(\begin{array}{cc}
U & 0 \\
0 & \mathbb{I}
\end{array}\right) W=\frac{1}{2}\left(\begin{array}{cc}
U+\mathbb{I} & i(U-\mathbb{I}) \\
-i(U-\mathbb{I}) & U+\mathbb{I}
\end{array}\right)
$$

where $U$ is a unitary matrix.

\section{Extension theory}

Here we consider the extension theory for a symmetric operator $\mathcal{L}_{0}$ on a Hilbert space [2, 3, 13, 14]. First we recall some well known facts from operator theory. The domain of the adjoint operator $\mathcal{L}_{0}^{\star}$ can be expressed

$$
\operatorname{Dom}\left(\mathcal{L}_{0}^{\star}\right)=\operatorname{Dom}\left(\mathcal{L}_{0}\right)+\mathcal{N}_{+i}+\mathcal{N}_{-i}
$$

where these three subspaces are linearly independent. The eigenspaces

$$
\mathcal{N}_{ \pm i} \equiv \operatorname{ker}\left(\mathcal{L}_{0}^{\star} \pm i\right)
$$

are known as the deficiency subspaces and the deficiency indices $\left(n_{+}, n_{-}\right)$are the dimensions of the deficiency subspaces $n_{ \pm} \equiv \operatorname{dim} \mathcal{N}_{ \pm i}$. In what follows we assume $n_{ \pm}<\infty$.

Typically, the extensions of $\mathcal{L}_{0}$ are specified by a unitary map between the deficiency subspaces [2, 14] and self-adjoint extensions of $\mathcal{L}_{0}$ exist when $n_{+}=n_{-}$. Alternatively, extensions may be described by consideration of the boundary form

$$
\mathcal{J}(f, g) \equiv\left(\mathcal{L}_{0}^{\star} f, g\right)-\left(f, \mathcal{L}_{0}^{\star} g\right),
$$

where $f, g \in \operatorname{Dom}\left(\mathcal{L}_{0}^{\star}\right)$ - see [13] for a detailed account. The boundary form $\mathcal{J}(\cdot, \cdot)$ is actually a hermitian symplectic form and when restricted to $\mathcal{N}_{+i}+\mathcal{N}_{-i}$ is nondegenerate, defining a hermitian symplectic space (the form is degenerate on $\operatorname{Dom}\left(\mathcal{L}_{0}\right)$, a simple consequence of the fact that $\mathcal{L}_{0}$ is symmetric).

Proposition 1 The hermitian symplectic space formed by the boundary form $\mathcal{J}$ on $\mathcal{N}_{+i}+\mathcal{N}_{-i}$ is characterised, in the sense of lemma 1, by the deficiency indices $n_{ \pm}$. 
Proof: Suppose that we have orthonormal bases $\left\{f_{+, i}\right\}_{i=1}^{n_{+}},\left\{f_{-, i}\right\}_{i=1}^{n_{-}}$for $\mathcal{N}_{+i}$ and $\mathcal{N}_{-i}$ respectively. We use these bases to write the boundary form as a matrix

$$
\omega_{i j}=\mathcal{J}\left(f_{-, i}, f_{-, j}\right)=-2 i \delta_{i j} .
$$

This completes the proof.

In terms of this hermitian symplectic space it is not difficult to see that the extensions of $\mathcal{L}_{0}$ correspond to isotropic subspaces and, when the space is canonical (ie. $n_{+}=n_{-}$), that the self-adjoint extensions correspond to Lagrange planes.

\subsection{The Schrödinger operator on the graph with trivial com- pact part}

Here we consider the non-compact graph consisting of $n$ semi-axes connected at a single vertex, we denote such a graph by $\Gamma_{n}$. Functions on $\Gamma_{n}$ may be represented by elements of the Hilbert space

$$
H\left(\Gamma_{n}\right)=\oplus_{i=1}^{n} L^{2}([0, \infty)) .
$$

The elements of $H\left(\Gamma_{n}\right)$ are $n$-dimensional vector functions and the inner product on $H\left(\Gamma_{n}\right)$ is

$$
(\phi, \psi)=\sum_{i=1}^{n}\left(\phi_{i}, \psi_{i}\right)_{L^{2}([0, \infty))}=\sum_{i=1}^{n} \int_{0}^{\infty} \bar{\phi}_{i}(x) \psi_{i}(x) d x
$$

where $\phi_{i}$ are the components of $\phi$.

Let us consider the symmetric Schrödinger operator, $\mathcal{L}_{0}$ in $H\left(\Gamma_{n}\right)$ which acts on components by

$$
\mathcal{L}_{0} \psi_{i} \equiv-\frac{d^{2} \psi_{i}}{d x_{i}^{2}}+q_{i} \psi_{i},
$$

and has domain consisting of the smooth functions with compact suppport in the open interval

$$
D\left(\mathcal{L}_{0}\right)=\oplus_{i=1}^{n} C_{0}^{\infty}((0, \infty)) .
$$

The potentials $q_{i}$ are supposed to be continuous real valued functions which are integrable with finite first moment, ie.

$$
\int_{0}^{\infty}(1+x)\left|q_{i}(x)\right| d x<\infty .
$$

It is easy to see that the deficiency indices of $\mathcal{L}_{0}$ are $(n, n)$. Consequently we may consider the self-adjoint extensions of $\mathcal{L}_{0}$ and indeed, using the results of Neumann extension theory [2] parameterise these extensions by the unitary matrices $\mathrm{U}(n)$.

The problem of finding self-adjoint boundary conditions for such an operator is discussed in detail in [10, 6]. In [10] all self-adjoint boundary conditions are parameterised non-uniquely in terms of two $n$-th order matrices, $A B$, 
such that $(A B)$ is of maximal rank and $A B^{\star}=B A^{\star}$ is hermitian (in this paper the authors consider graphs with trivial compact part as well as graphs with non-trivial compact part).

Instead, here we will use the discussion of hermitian symplectic spaces to parameterise all of the self-adjoint boundary conditions at the origin in terms of a unitary matrix $U$. A simple calculation using integration by parts shows that the boundary form for the Schrödinger operator is

$$
\left(\mathcal{L}_{0}^{\star} \psi, \phi\right)-\left(\psi, \mathcal{L}_{0}^{\star} \phi\right)=\left.\sum_{j=1}^{n}\left[\bar{\psi}_{i} \phi_{i, x}-\bar{\psi}_{i, x} \phi_{i}\right]\right|_{0} .
$$

This boundary form may be thought of as acting in the $2 n$-dimensional hermitian symplectic space, $H_{2 n}$, of boundary values at the origin. The boundary form can be written

$$
\mathcal{J}(\psi, \phi)=(\psi, J \phi)
$$

where on the right hand side we use the inner product in $\mathbb{C}^{2 n}$ and $\psi, \phi$ are vectors in $\mathbb{C}^{2 n}$ of the form

$$
\left(\left.\psi_{1}\right|_{0}, \ldots,\left.\psi_{n}\right|_{0},\left.\psi_{1, x}\right|_{0}, \ldots,\left.\psi_{n, x}\right|_{0}\right)^{T} .
$$

Consequently this defines a canonical basis. Let us represent the canonical basis elements explicitly as $\left\{\xi_{0, i}\right\}_{i=1}^{2 n} \in H_{2 n}$ where for $i=1, \ldots, n, \xi_{0, i}$ represents the boundary condition $\left.\psi_{i}\right|_{0}=1$; and for $i=n+1, \ldots, 2 n$ it represents the boundary condition $\left.\psi_{i, x}\right|_{0}=1$. The first $n$ and last $n$ elements of a canonical basis each span a Lagrange plane - the first $n$ basis vectors specify self-adjoint Neumann boundary conditions, and the last $n$ basis vectors specify self-adjoint Dirichlet boundary conditions.

We fix a unitary matrix $U$ and consider the associated self-adjoint boundary conditions specifying a Lagrange plane. From corollary 2 the basis for the Lagrange plane defined by $U$ is given by

$$
\xi_{i}=\sum_{j=1}^{2 n} g_{i j} \xi_{0, j} \quad \text { for } i=1, \ldots, n,
$$

where $g$ is defined by equation (77). Writing this in terms of boundary values we see that (up to a transposition) the set of self-adjoint boundary values is

$$
\left(\left.\psi_{1}\right|_{0}, \ldots,\left.\psi_{n}\right|_{0},\left.\psi_{i, x}\right|_{0}, \ldots,\left.\psi_{n, x}\right|_{0}\right)^{T} \in \operatorname{Ran}\left(\begin{array}{c}
\frac{1}{2}(U+\mathbb{I}) \\
\frac{i}{2}(U-\mathbb{I})
\end{array}\right) .
$$

It is convenient to have the self-adjoint boundary conditions, ie. to have an expression in terms of the kernel rather than the range of a matrix. This is possible if we note that

$$
\operatorname{Ran}\left(\begin{array}{c}
\frac{1}{2}(U+\mathbb{I}) \\
\frac{i}{2}(U-\mathbb{I})
\end{array}\right)=\operatorname{ker}\left(\frac{i}{2}\left(U^{\star}-\mathbb{I}\right), \frac{1}{2}\left(U^{\star}+\mathbb{I}\right)\right)
$$


which follows from equation (51) and the fact that both of these matrices are of rank $n$. Consequently, the boundary conditions may be expressed

$$
\left.\frac{i}{2}\left(U^{\star}-\mathbb{I}\right) \psi\right|_{0}+\left.\frac{1}{2}\left(U^{\star}+\mathbb{I}\right) \psi_{x}\right|_{0}=0 .
$$

In the remainder of this subsection we will discuss how the matrix $U$, used to describe the boundary conditions, appears in the asymptotics of the scattering matrix. It is convenient to consider the Schrödinger operator on the graph with $n$ rays as a matrix operator, with diagonal potential, see [8, 9]. Let us consider the matrix of $n$ solutions of Schrödinger equation $\mathcal{L} \Xi=\lambda \Xi$ on the graph satisfying the following boundary conditions at the origin

$$
\left.\Xi\right|_{0}=\frac{1}{2}(U+\mathbb{I}) \equiv A,\left.\quad \Xi_{x}\right|_{0}=\frac{i}{2}(U-\mathbb{I}) \equiv B .
$$

It is clear, from equation (15), that each column of $\Xi$ satisfies the self-adjoint boundary conditions, ie. equation (11), and hence is (formally) an eigenfunction of the self-adjoint Schrödinger operator on the graph with boundary conditions prescribed by $U$.

Likewise we can define the Jost solutions, $F_{ \pm}$, as the matrix of solutions of the homogeneous equation $\mathcal{L} F_{ \pm}=\lambda F_{ \pm}$, with asymptotic behaviour

$$
\lim _{x \rightarrow \infty} F_{ \pm}(x, k) \sim e^{ \pm i k x} \mathbb{I} .
$$

We denote $\lambda=k^{2}$. As the Jost solutions form a complete set of solutions we can write

$$
\Xi(x, k)=F_{-}(x, k) M_{-}(k)+F_{+}(x, k) M_{+}(k) .
$$

In this notation we define the scattering wave solutions

$$
\Psi(x, k) \equiv \Xi(x, k) M_{-}^{-1}=F_{-}+F_{+} S(k)
$$

where $S(k)$ is known as the scattering matrix. The coefficients $M_{ \pm}$can be evaluated by taking the Wronskian of $\Xi$ and $F_{+}$or $F_{-}[8$

$$
M_{ \pm}= \pm \frac{1}{2 i k}\left[\mathrm{~F}_{ \pm}^{\dagger} B-\mathrm{F}_{ \pm, x}^{\dagger} A\right] .
$$

where $\mathrm{F}_{ \pm}(k) \equiv F_{ \pm}(0, k)$ are known as the Jost functions and ${ }^{\dagger}$ is the involution $Y^{\dagger}(x, k) \equiv Y^{\star}(x, \bar{k})$. The Wronskian of $\Xi^{\dagger}$ and $\Xi$

$$
W\left\{\Xi^{\dagger}, \Xi\right\}=\left.\left[\Xi^{\dagger} \Xi_{x}-\Xi_{x}^{\dagger} \Xi\right]\right|_{0}=A^{\star} B-B^{\star} A=0,
$$

is always zero. Moreover, if we write $\Xi$ in terms of the scattering wave solutions

$$
W\left\{\Xi^{\dagger}, \Xi\right\}=M_{-}^{\dagger} W\left\{F_{-}^{\dagger}+S^{\dagger} F_{+}^{\dagger}, F_{-}+F_{+} S\right\} M_{-}=2 i k M_{-}^{\dagger}\left[-\mathbb{I}+S^{\dagger} S\right] M_{-},
$$

we see, since $S^{\dagger}=S^{\star}$ for $k \in \mathbb{R}$, that the scattering matrix is unitary for real $k$.

If we diagonalise $U$, and use the well known asympototics of the Jost functions [1, 8] in the above expression for $M_{ \pm}$, we see that the scattering matrix has the following asymptotic behaviour: 
Lemma 8 Given the self-adjoint operator $\mathcal{L}$, with associated unitary matrix $U$ defining the boundary conditions of $\mathcal{L}$, the scattering matrix of $\mathcal{L}$ has the asymptotics

$$
\lim _{k \rightarrow \infty} S(k) \sim \hat{U}
$$

where $\hat{U}$ is a unitary hermitian matrix derived from $U$ by applying the map

$$
z \mapsto\left\{\begin{array}{cc}
1 & : z \in \mathbb{T} \backslash\{-1\} \\
-1 & : z=-1
\end{array}\right.
$$

to the spectrum of $U$. Here $\mathbb{T}$ is the unit circle in $\mathbb{C}$.

Proof: Let us diagonalise the matrix $U$. In this basis, using equation (14) and the asymptotics of the Jost functions, the scattering matrix approaches

$$
\lim _{k \rightarrow \infty}-\left[\left(e^{i \varphi_{j}}-1\right)+k\left(e^{i \varphi_{j}}+1\right)\right]\left[\left(e^{i \varphi_{j}}-1\right)-k\left(e^{i \varphi_{j}}+1\right)\right]^{-1}
$$

in the limit of large $k$. Here the $e^{i \varphi_{j}}$ are the unitary eigenvalues of $U$. There are two cases; when $e^{i \varphi_{j}}=-1$, this limit is -1 , and when $e^{i \varphi_{j}} \neq-1$ the limit is 1 .

We note that those boundary conditions which are defined by unitary matrices which in addition are hermitian matrices can be expressed by projections - the terms $\frac{1}{2}(U \pm \mathbb{I})$ are really orthogonal projections

$$
P=\frac{1}{2}(U+\mathbb{I}), \quad P^{\perp}=\mathbb{I}-P=-\frac{1}{2}(U-\mathbb{I}) .
$$

which follows simply from the fact that $U=U^{\star}=U^{-1}$. Using this notation and orthogonality we can write the boundary conditions, equation (11), as

$$
\left.P^{\perp} \psi\right|_{0}=0,\left.\quad P \psi_{x}\right|_{0}=0 .
$$

Consequently these boundary conditions are characterised by the fact that the conditions on the functions and the derivatives of the functions at the origin are independently specified.

The associated scattering matrix has the form

$$
S(k)=-\left[i \mathrm{~F}_{+}^{\dagger} P^{\perp}+\mathrm{F}_{+, x}^{\dagger} P\right]\left[i \mathrm{~F}_{-}^{\dagger} P^{\perp}+\mathrm{F}_{-, x}^{\dagger} P\right]^{-1} .
$$

In the case of zero potential so that the Jost solutions are exponential functions we see that the scattering matrix is constant

$$
S(k)=-\left[P^{\perp}-k P\right]\left[P^{\perp}+k P\right]^{-1}=-P^{\perp}+P=U .
$$

Therefore the scattering wave has no poles and there are no discrete eigenvalues.

In contrast if $U$ is not hermitian we will have discrete eigenvalues, or alternatively resonances, when the potential is, apart from at the origin, identically zero. This reproduces all cases-like for instance a $\delta$ or $\delta^{\prime}$-interaction at the origin - in which bound states or resonances appear for a zero-range potential. 


\section{Acknowledgements}

The author would like to thank Prof B.S. Pavlov for his advice and many useful conversations.

\section{References}

[1] Z. S. Agranovich and V. A. Marchenko. The Inverse Problem of Scattering Theory. Gordon and Breach, New York, 1963.

[2] N. I. Akhiezer and I. M. Glazman. Theory of Linear Operators in Hilbert Space. Frederick Ungar Publishing, New York, 1966.

[3] S. Albeverio and P. Kurasov. Singular Perturbations of Differential Operators. London Mathematical Society Lecture Note Series 271. Cambridge University Press, Cambridge, 2000.

[4] V. I. Arnold. Mathematical Methods of Classical Mechanics. Graduate Texts in Mathematics 60. Springer, Berlin, 1978.

[5] V. I. Arnold and A. B. Givental. Symplectic geometry. In V. I. Arnold and S. P. Novikov, editors, Dynamical Systems IV. Springer, Berlin, 1990.

[6] P. Exner and P. S̆eba. Free quantum motion on a branching graph. Rep. Math. Phys, 28:7-26, 1989.

[7] A. T. Fomenko. Symplectic Geometry. Gordon and Breach, 1988.

[8] M. Harmer. The Matrix Schrödinger Operator and Schrödinger Operator on Graphs. PhD thesis, University of Auckland, 2000.

[9] M. Harmer. Inverse scattering for the matrix Schrödinger operator and Schrödinger operator on graphs with general self-adjoint boundary conditions. ANZIAM Journal, 43:1-8, 2002.

[10] V. Kostrykin and R. Schrader. Kirchhoff's rule for quantum wires. J. Phys A: Math. Gen., 32:595-630, 1999.

[11] J. E. Marsden and T. S. Ratiu. Introduction to Mechanics and Symmetry. Springer, New York, 1994.

[12] S. P. Novikov. Schrödinger operators on graphs and symplectic geometry. In E. Bierstone, B. Khesin, A. Khovanskii, and J. Marsden, editors, The Arnol'dfest (Toronto, ON, 1997), volume 24 of Fields Institute Communications, pages 397-413, 1999.

[13] B. S. Pavlov. The theory of extensions and explicitly solvable models. Uspekhi Math. Nauk-Russian. Math. Surveys, 42(6):127-168, 1987.

[14] M. Reed and B. Simon. Methods of Modern Mathematical Physics. Academic Press, New York, 1972. 